\title{
Neuronal transcription factors in lifespan control
}

\author{
Alexander Symonenko \\ IMG RAS, Moscow, Russia \\ symonenko@gmail.com
}

\author{
Natalia Roshina \\ IMG RAS, Moscow, Russia \\ VIGG RAS, Mosocw, Russia \\ nwumr@yandex.ru
}

\author{
Anna Krementsova \\ IMG RAS, Moscow, Russia \\ IBCP RAS, Moscow, Russia \\ akrementsova@mail.ru
}

Elena Pasyukova

IMG RAS, Moscow, Russia egpas@rambler.ru

\begin{abstract}
In D. melanogaster, neuronal transcription factors were shown to participate in lifespan control. To identify common pathways for regulating neuronal properties and longevity, we examined molecular targets for transcription factor Stc. Among the targets, genes (proteins) involved in the control of neurogenesis, chromatin structure, and energy metabolism were identified. Our results allowed us to suggest several possible molecular mechanisms by which Stc is involved in lifespan control.
\end{abstract}

Keywords - lifespan, transcription, factors, neurons, Drosophila

Motivation and aim

\section{Motivation}

Transcription factors play an important role in the systemic control of gene expression. The role of transcriptional cascades in the regulation of neurogenesis in D. melanogaster is well known [1]. Earlier, we showed that a number of genes (stc, Lim3, esg, and others) encoding neuronal transcription factors are involved in controlling lifespan [2-4].

Aim

In order to understand which systemic molecular and genetic mechanisms determine the effect of the transcription factor Stc on lifespan and how they are associated with the control of the neuronal development and function, we examined Stc target genes and regulatory cascades in which it is involved.

\section{Methods}

Regular RNA-seq and Real-time RT-qPCR analyses were used to experimentally assess stc targets. To build stc interaction networks, we used data from the open repositories: Flybase (http://flybase.org/), Flymine

(https://www.flymine.org/) and BioGRID (https://thebiogrid.org/). To build the networks, the esyN toolkit (easy networks, http://www.esyn.org/ ) was used.

\section{Results}

The stc gene encodes a transcription factor of RNA polymerase II homologous to human transcription factor NFX1 [5]. We found that Stc might impact the transcription levels of the $E(b x)$ gene, which encodes the factor of the nucleosome remodeling complex, and interacts with the Ada2b protein, a component of the multi-subunit SAGA complex, which affects the level of histone acetylation, and thus the chromatin structure. This result reinforces our earlier assumption about the possibility of epigenetic inheritance of properties caused by changes in stc expression at the embryonic stage over a number of cell generations. Stc also interacted with the genes encoding the SERCA and THADA proteins that control the body's temperature homeostasis, both in human and drosophila models, as well as with other genes that control energy metabolism. The functional variability of SERCA and THADA may have important adaptive value in populations [6]. As expected, Stc interacted with genes involved in neurogenesis. The most interesting interactions were confirmed by data on the effects of stc (Stc) on energy metabolism and neuronal structure and function.

Our results allowed us to identify several possible molecular mechanisms by which stc (Stc) is involved in lifespan control. However, data obtained so far did not indicate common regulatory pathways by which stc (Stc) might control neuronal functions and lifespan.

ACKNOWLEDGMENT

Supported by the RFBR (15-04-05797a, 18-04-01127-a) and by RF Budget (AAAA-A19-119022590053-3).

\section{REFERENCES}

[1] Jothi R. et al. (2009) Genomic analysis reveals a tight link between transcription factor dynamics and regulatory network architecture. Mol Syst. Biol. 5:294.

[2] Roshina N.V. et al. (2014) Embryonic expression of shuttle craft, a Drosophila gene involved in neuron development, is associated with adult lifespan. Aging US 6:1076-1093.

[3] Rybina O.Y. et al. (2017) Tissue-specific transcription of the neuronal gene Lim3 affects Drosophila melanogaster lifespan and locomotion. Biogerontology 18:739-757.

[4] Symonenko A.V. et al. (2018) Reduced neuronal transcription of escargot, the Drosophila gene encoding a Snail-type transcription factor, promotes longevity. Front. Genet. 9:151.

[5] Stroumbakis N.D. et al. (1996) A homolog of human transcription factor NF-X1 encoded by the Drosophila shuttle craft gene is required in the embryonic central nervous system. Mol. Cell. Biol. 16:192-201.

[6] Moraru A. et al. (2017) THADA regulates the organismal balance between energy storage and heat production. Dev. Cell 41:72-81. 